\begin{tabular}{|c|c|}
\hline \multicolumn{2}{|c|}{ Statistica Sinica Preprint No: SS-2021-0238 } \\
\hline Title & Bayesian Predictive Inference Without a Prior \\
\hline Manuscript ID & SS-2021-0238 \\
\hline URL & http://www.stat.sinica.edu.tw/statistica/ \\
\hline DOI & $10.5705 /$ ss.202021.0238 \\
\hline Complete List of Authors & $\begin{array}{l}\text { Patrizia Berti, } \\
\text { Emanuela Dreassi, } \\
\text { Fabrizio Leisen, } \\
\text { Luca Pratelli and } \\
\text { Pietro Rigo }\end{array}$ \\
\hline Corresponding Author & Pietro Rigo \\
\hline E-mail & pietro.rigo@unibo.it \\
\hline Notice: Accepted version subje & ct to English editing. \\
\hline
\end{tabular}


Statistica Sinica

\title{
BAYESIAN PREDICTIVE INFERENCE WITHOUT A PRIOR
}

\author{
Patrizia Berti ${ }^{1}$, Emanuela Dreassi ${ }^{2}$, Fabrizio Leisen $^{3}$, \\ Luca Pratelli ${ }^{4}$ and Pietro Rigo ${ }^{5}$ \\ ${ }^{1}$ Università di Modena e Reggio-Emilia, ${ }^{2}$ Università di Firenze, \\ ${ }^{3}$ University of Nottingham, ${ }^{4}$ Accademia Navale di Livorno and \\ ${ }^{5}$ Università di Bologna
}

Abstract: Let $\left(X_{n}: n \geq 1\right)$ be a sequence of random observations. Let $\sigma_{n}(\cdot)=$ $P\left(X_{n+1} \in \cdot \mid X_{1}, \ldots, X_{n}\right)$ be the $n$-th predictive distribution and $\sigma_{0}(\cdot)=P\left(X_{1} \in \cdot\right)$ the marginal distribution of $X_{1}$. To make predictions on $\left(X_{n}\right)$, a Bayesian forecaster only needs the collection $\sigma=\left(\sigma_{n}: n \geq 0\right)$. Because of the IonescuTulcea theorem, $\sigma$ can be assigned directly, without passing through the usual prior/posterior scheme. One main advantage is that no prior probability has to be selected. This point of view is adopted in this paper. The choice of $\sigma$ is only subjected to two requirements: (i) The resulting sequence $\left(X_{n}\right)$ is conditionally identically distributed, in the sense of [4]; (ii) Each $\sigma_{n+1}$ is a simple recursive update of $\sigma_{n}$. Various new $\sigma$ satisfying (i)-(ii) are introduced and investigated. For such $\sigma$, the asymptotics of $\sigma_{n}$, as $n \rightarrow \infty$, is determined. In some cases, the probability distribution of $\left(X_{n}\right)$ is also evaluated. 
Key words and phrases: Asymptotics, Bayesian nonparametrics, Conditional identity in distribution, Exchangeability, Predictive distribution, Sequential prediction, Total variation distance.

MSC 2020 subject classifications: 62F15, 62M20, 60G25, 60G09.

\section{Introduction}

Consider a Bayesian forecaster who makes predictions on a sequence $\left(X_{n}: n \geq 1\right)$

of random observations. At each time $n$, she aims to predict $X_{n+1}$ based on $\left(X_{1}, \ldots, X_{n}\right)$. To this end, she needs to assign the conditional distribution of $X_{n+1}$ given $\left(X_{1}, \ldots, X_{n}\right)$, usually called the $n$-th predictive distribution.

To formalize this problem, we fix a measurable space $(S, \mathcal{B})$ and we take $X_{n}$ to be the $n$-th coordinate random variable on $S^{\infty}$, namely

$$
X_{n}\left(s_{1}, \ldots, s_{n}, \ldots\right)=s_{n} \quad \text { for all } n \geq 1 \text { and }\left(s_{1}, \ldots, s_{n}, \ldots\right) \in S^{\infty}
$$

To avoid technicalities, we assume that $S$ is a Borel subset of a Polish space and $\mathcal{B}$ is the Borel $\sigma$-field on $S$. Moreover, following Dubins and Savage [14], we introduce the notion of strategy.

Let $\mathcal{P}$ denote the collection of all probability measures on $\mathcal{B}$. A strategy is a sequence $\sigma=\left(\sigma_{0}, \sigma_{1}, \ldots\right)$ such that

- $\sigma_{0} \in \mathcal{P}$ and $\sigma_{n}=\left\{\sigma_{n}(x): x \in S^{n}\right\}$ is a collection of elements of $\mathcal{P}$;

- The map $x \mapsto \sigma_{n}(x)(A)$ is $\mathcal{B}^{n}$-measurable for fixed $n \geq 1$ and $A \in \mathcal{B}$. 
Here, $\sigma_{0}$ should be regarded as the marginal distribution of $X_{1}$ and $\sigma_{n}(x)$ as the conditional distribution of $X_{n+1}$ given that $\left(X_{1}, \ldots, X_{n}\right)=x$. Moreover, $\sigma_{n}(x)(A)$ denotes the probability attached to the event $A$ by the probability measure $\sigma_{n}(x)$.

An important special case is when the strategy $\sigma$ is dominated by a fixed measure $\lambda$ on $(S, \mathcal{B})$. This means that $\sigma_{n}(x)$ has a density with respect to $\lambda$, say $f_{n}(\cdot \mid x)$, for all $n$ and $x$. Hence, $\sigma_{n}(x)$ can be written as

$$
\sigma_{n}(x)(A)=\int_{A} f_{n}(z \mid x) \lambda(d z) \quad \text { for all } A \in \mathcal{B}
$$

For instance, if $S$ is countable, any strategy $\sigma$ is dominated by $\lambda=$ counting measure. Or else, if $S=\mathbb{R}$, some meaningful strategies are dominated by $\lambda=$ Lebesgue measure. Clearly, in the dominated case, the strategy $\sigma$ can be identified with the sequence $\left(f_{0}, f_{1}, \ldots\right)$ of predictive densities. However, in this paper, we deal with general strategies and dominated strategies are just a (remarkable) special case.

For any strategy $\sigma$ (dominated or not), there is a unique probability measure $P_{\sigma}$ on $\left(S^{\infty}, \mathcal{B}^{\infty}\right)$ such that

$$
\begin{gathered}
P_{\sigma}\left(X_{1} \in \cdot\right)=\sigma_{0} \text { and } P_{\sigma}\left(X_{n+1} \in \cdot \mid\left(X_{1}, \ldots, X_{n}\right)=x\right)=\sigma_{n}(x) \\
\text { for all } n \geq 1 \text { and } P_{\sigma} \text {-almost all } x \in S^{n} .
\end{gathered}
$$

The above result, due to Ionescu-Tulcea, provides the theoretical foundations of Bayesian predictive inference. To make predictions on $\left(X_{n}\right)$, 
1.1 Motivations

one needs precisely a strategy $\sigma$. The Ionescu-Tulcea theorem guarantees that, for any $\sigma$, the predictions based on $\sigma$ are consistent with a unique probability distribution $P_{\sigma}$ for the data sequence $\left(X_{n}\right)$.

However, $\left(X_{n}\right)$ is usually required some distributional properties suggested by the specific problem under consideration. For instance, $\left(X_{n}\right)$ is asked to be exchangeable, or stationary, or Markov, and so on. In these cases, the strategy $\sigma$ can not be arbitrary, for $P_{\sigma}$ must belong to some given class of probability measures on $\left(S^{\infty}, \mathcal{B}^{\infty}\right)$.

\subsection{Motivations}

In a Bayesian framework, $\left(X_{n}\right)$ is typically assumed to be exchangeable. In that case, there are essentially two approaches for selecting a strategy $\sigma$. For definiteness, as in [8], we call them the standard approach (SA) and the non-standard approach (NSA). Both are admissible, from the Bayesian point of view, and both lead to a full specification of the probability distribution of $\left(X_{n}\right)$.

According to SA, to obtain $\sigma$, one should:

- Select a prior $\pi$, namely, a probability measure on $\mathcal{P}$;

- Calculate the posterior of $\pi$ given that $\left(X_{1}, \ldots, X_{n}\right)=x$, say $\pi_{n}(x)$; 
1.1 Motivations

- Evaluate $\sigma$ as

$$
\sigma_{n}(x)(A)=\int_{\mathcal{P}} p(A) \pi_{n}(x)(d p) \quad \text { for all } A \in \mathcal{B}
$$

where $\pi_{0}(x)$ is meant as $\pi_{0}(x)=\pi$.

Instead, according to NSA, the strategy $\sigma$ can be assigned directly, without passing through the above prior/posterior scheme. Rather than choosing $\pi$ and evaluating $\pi_{n}$ and $\sigma_{n}$, the forecaster merely selects her predictive $\sigma_{n}$. This procedure makes sense because of the Ionescu-Tulcea theorem. See e.g. [3], [5], [8], [9], [12], [15], [16], [17], [19], [20], [21], [23], [25], 26].

The merits and drawbacks of $\mathrm{SA}$ and NSA are discussed in [8]. In short, SA is a cornerstone of Bayesian inference but is not motivated by prediction alone. Its main scope is to make inference on other features of the data distribution, such as a random parameter (possibly, infinite dimensional). However, when prediction is the main target, SA is clearly involved. In turn, NSA has essentially four merits.

- NSA requires the assignment of probabilities on observable facts only. The next observation $X_{n+1}$ is actually observable, while $\pi$ and $\pi_{n}$ (being probabilities on $\mathcal{P}$ ) do not deal with observable facts.

- The data sequence $\left(X_{n}\right)$ is not forced to satisfy any distributional assumption. In particular, $\left(X_{n}\right)$ may fail to be exchangeable. 
1.1 Motivations

- The strategy $\sigma$ may be assigned stepwise. At each time $n$, the forecaster has observed $x=\left(x_{1}, \ldots, x_{n}\right) \in S^{n}$ and has already selected $\sigma_{0}, \sigma_{1}\left(x_{1}\right), \ldots, \sigma_{n-1}\left(x_{1}, \ldots, x_{n-1}\right)$. Then, to predict $X_{n+1}$, she is still free to select $\sigma_{n}(x)$ as she wants. No choice of $\sigma_{n}(x)$ is precluded. According to us, this is consistent with the Bayesian view, where the observed data are fixed and one should condition on them. A similar point of view is highlighted in [15].

- NSA is more straightforward than SA when prediction is the main goal. In this case, why select the prior $\pi$ explicitly ? Rather than wondering about $\pi$, it seems reasonable to reflect on how $X_{n+1}$ is affected by $\left(X_{1}, \ldots, X_{n}\right)$.

The above remarks refer to any (Bayesian) prediction problem, both parametric and nonparametric. However, NSA is especially appealing in the nonparametric case, where selecting a prior with large support is usually hard. For instance, NSA is quite natural when dealing with species sampling sequences. Indeed, this paper has been written having the nonparametric framework in mind.

If $\left(X_{n}\right)$ is assumed to be exchangeable, however, NSA has a drawback. To apply NSA with exchangeable data, one should first characterize those strategies $\sigma$ which make $\left(X_{n}\right)$ exchangeable under $P_{\sigma}$. A nice characterization is [16, Th. 3.1]. However, the conditions on $\sigma$ for making $\left(X_{n}\right)$ 
1.1 Motivations

exchangeable are quite hard to check in real problems.

To bypass this drawback, the exchangeability assumption could be weakened. One option is to assume $\left(X_{n}\right)$ conditionally identically distributed (c.i.d.). We refer to Subsection 2.2 for c.i.d. sequences. Here, we just mention a few reasons for taking c.i.d. data into account.

- Essentially, $\left(X_{n}\right)$ is c.i.d. if, at each time $n$, the future observations $\left(X_{k}: k>n\right)$ are identically distributed given the past $\left(X_{1}, \ldots, X_{n}\right)$. This assumption is quite natural in several prediction problems.

- The asymptotic behavior of c.i.d. sequences is very similar to that of exchangeable ones.

- A meaningful part of the usual Bayesian machinery can be developed under the sole assumption that $\left(X_{n}\right)$ is c.i.d.; see [15].

- A number of interesting strategies cannot be used if $\left(X_{n}\right)$ is asked to be exchangeable, but are available if $\left(X_{n}\right)$ is only required to be c.i.d.; see e.g. [8]. Furthermore, conditional identity in distribution is more reasonable than exchangeability in a few real problems. Examples occur in various fields, including clinical trials, generalized Polya urns, species sampling models and disease surveillance; see [1], 2], [4], [11].

- It is not hard to characterize the strategies $\sigma$ which make $\left(X_{n}\right)$ c.i.d. under $P_{\sigma}$; see Theorem 1. Therefore, unlike the exchangeable case, 


\subsection{Our contribution}

NSA can be easily implemented.

\subsection{Our contribution}

This paper aims to develop NSA for c.i.d. data. It is the natural follow up of [8] but all results and examples (with the only exception of Example 3) are new. Our main goal is to introduce and investigate new strategies $\sigma$ having the following two properties:

(i) The sequence $\left(X_{n}\right)$ is c.i.d. under $P_{\sigma}$;

(ii) $\sigma_{n+1}$ is a simple recursive update of $\sigma_{n}$ for each $n \geq 0$.

Condition (i) has been already discussed. Condition (ii) is to obtain a fast online Bayesian prediction, in the spirit of [20]. Ideally, condition (ii) should imply that each predictive can be evaluated through a simple recursion on the previous one.

To make some examples, for all $x=\left(x_{1}, \ldots, x_{n}\right) \in S^{n}$ and $y \in S$, write

$$
(x, y)=\left(x_{1}, \ldots, x_{n}, y\right)
$$

In this notation, $(x, y)$ is a point of $S^{n+1}, x$ is the sub-vector containing the first $n$ coordinates and $y$ is the $(n+1)$-th coordinate. Then, for instance, condition (ii) holds if $\sigma$ satisfies the recursive equations

$$
\sigma_{0}=\alpha_{0} \quad \text { and } \quad \sigma_{n+1}(x, y)=q_{n}(x) \sigma_{n}(x)+\left(1-q_{n}(x)\right) \alpha_{n+1}(x, y)
$$




\subsection{Our contribution}

for all $n \geq 0, x \in S^{n}$ and $y \in S$, where $q_{n}: S^{n} \rightarrow[0,1]$ is any measurable function and $\alpha=\left(\alpha_{0}, \alpha_{1}, \ldots\right)$ is a given strategy.

According to (1.1), the predictive $\sigma_{n+1}(x, y)$ is a convex combination of the previous predictive $\sigma_{n}(x)$ and the new contribution $\alpha_{n+1}(x, y)$, with a weight $q_{n}(x)$ not depending on the last observation $y$. A possible interpretation is that, at time $n+1$, after observing $(x, y)$, the next observation is drawn from $\sigma_{n}(x)$ with probability $q_{n}(x)$ or from $\alpha_{n+1}(x, y)$ with probability $1-q_{n}(x)$. Even if simple, this updating rule is able to model various real situations; see Examples 1-9. Moreover, no prior probability is required. The forecaster has only to choose the weights $q_{0}, q_{1}, \ldots$ and the strategy $\alpha$.

An obvious criticism to 1.1 is that, to calculate $\sigma$, the forecaster should first select another strategy $\alpha$ (in addition to the weights $q_{0}, q_{1}, \ldots$ ). And, in general, choosing $\alpha$ is as difficult as choosing $\sigma$. This is only partially true, for the choice of $\alpha$ is not so hard in several real situations. Exploiting an idea from [20], for instance, $\alpha$ can be obtained via copulas; see Example 1. Or else, $\alpha$ can be built by iterated conditioning; see Example 2, More importantly, the choice of $\alpha$ is simpler in the Markovian case. In this paper, a strategy $\alpha$ is said to be Markovian if

$$
\alpha_{n}(x, y)=\alpha_{n}^{*}(y) \quad \text { for all } n \geq 2, x \in S^{n-1} \text { and } y \in S
$$

where $\alpha_{n}^{*}: S \rightarrow \mathcal{P}$ is any measurable map. With a slight abuse of notation, when $\alpha$ is Markovian, we will write $\alpha_{n}(y)$ instead of $\alpha_{n}^{*}(y)$. 
1.2 Our contribution

In addition to (ii), $\sigma$ is required to satisfy condition (i). Our first result is that, if $\sigma$ satisfies $(1.1)$, then $\left(X_{n}\right)$ is c.i.d. under $P_{\sigma}$ provided $\sigma_{n}(x)(A)=\int \alpha_{n+1}(x, y)(A) \sigma_{n}(x)(d y) \quad$ for all $n \geq 0, x \in S^{n}$ and $A \in \mathcal{B}$

Such a condition becomes simpler if $\alpha$ is Markovian. Suppose indeed $\alpha$ is Markovian and recall that a filtration on $(S, \mathcal{B})$ is an increasing sequence $\mathcal{G}_{0} \subset \mathcal{G}_{1} \subset \ldots \subset \mathcal{B}$ of sub- $\sigma$-fields of $\mathcal{B}$. Then, $\left(X_{n}\right)$ is c.i.d. under $P_{\sigma}$ if $\alpha_{n+1}$ is the conditional distribution of $\alpha_{0}$ given $\mathcal{G}_{n}$ for all $n$ and some filtration $\left(\mathcal{G}_{n}\right)$. Formally,

$$
\alpha_{n+1}(\cdot)(A)=E_{\alpha_{0}}\left(1_{A} \mid \mathcal{G}_{n}\right), \quad \text { a.s. with respect to } \alpha_{0}
$$

for all $n \geq 0$, all $A \in \mathcal{B}$ and some filtration $\left(\mathcal{G}_{n}\right)$.

For instance, if $\mathcal{G}_{n}=\mathcal{B}$ for all $n$, condition $(1.2)$ yields $\alpha_{n+1}(y)=\delta_{y}$ for all $y \in S$ where $\delta_{y}$ denotes the unit mass at the point $y$. Indeed, some popular strategies admit representation (1.1) with $\alpha_{n+1}(y)=\delta_{y}$. Well known examples are Dirichlet sequences, Beta-GOS sequences, exponential smoothing and generalized Polya urns; see [1], [2] and [8, Sect. 4]. In all these cases, $\left(X_{n}\right)$ is c.i.d. under $P_{\sigma}$. At the opposite extreme, if $\mathcal{G}_{n}$ is the trivial $\sigma$-field for all $n$, condition (1.2) implies $\alpha_{n+1}(y)=\alpha_{0}$ for all $y \in S$. In this case, under $P_{\sigma},\left(X_{n}\right)$ is i.i.d. with common distribution $\alpha_{0}$.

More interestingly, take $\mathcal{G}_{n}$ to be the $\sigma$-field generated by a countable partition $\mathcal{H}_{n}$ of $S$, where $H \in \mathcal{B}$ and $\alpha_{0}(H)>0$ for all $H \in \mathcal{H}_{n}$. In this 
case, condition $(1.2)$ implies

$$
\alpha_{n+1}(y)=\sum_{H \in \mathcal{H}_{n}} 1_{H}(y) \alpha_{0}(\cdot \mid H)=\alpha_{0}\left(\cdot \mid H_{y}^{n}\right)
$$

where $H_{y}^{n}$ is the only $H \in \mathcal{H}_{n}$ such that $y \in H$. Moreover, $\mathcal{G}_{n} \subset \mathcal{G}_{n+1}$ if the partition $\mathcal{H}_{n+1}$ is finer than $\mathcal{H}_{n}$. With this choice of $\alpha$, several meaningful strategies satisfying (i)-(ii) can be obtained. For instance, if $q_{n}=(n+$ $c) /(n+1+c)$ for some constant $c>0$, one obtains

$$
\sigma_{n}(x)=\frac{c \alpha_{0}+\sum_{i=1}^{n} \alpha_{0}\left(\cdot \mid H_{x_{i}}^{i-1}\right)}{n+c}
$$

The above strategy $\sigma$ is analogous to that of a Dirichlet sequence, i.e.,

$$
\beta_{n}(x)=\frac{c \alpha_{0}+\sum_{i=1}^{n} \delta_{x_{i}}}{n+c}
$$

However, $\sigma$ and $\beta$ give rise to completely different behaviors for $\left(X_{n}\right)$. Firstly, $\left(X_{n}\right)$ is exchangeable under $P_{\beta}$ and only c.i.d. under $P_{\sigma}$. Secondly, if $G=\left\{X_{i}=X_{j}\right.$ for some $\left.i \neq j\right\}$, one obtains $P_{\sigma}(G)=0$ and $P_{\beta}(G)=1$ provided $\alpha_{0}$ is non-atomic. We also note that attaching probability zero to $G$ is useful in various applications.

This is just an example. Various other strategies come to the fore with a suitable choice of $\mathcal{H}_{n}$ and $q_{n}$; see Section 3 .

In addition to (1.1), a second class of strategies is introduced and investigated in this paper. Let $S=\mathbb{R}$ and $u_{n}$ a sequence of real numbers such 
that $0=u_{0}<u_{1}<u_{2}<\ldots<1$. Define $f_{0}(x)=0$ and

$$
f_{n+1}(x, y)=\sqrt{\frac{u_{n+1}-u_{n}}{1-u_{n}}} y+\left(1-\sqrt{\frac{u_{n+1}-u_{n}}{1-u_{n}}}\right) f_{n}(x)
$$

for all $n \geq 0, x \in S^{n}$ and $y \in S$. Define also a strategy $\sigma$ as

$$
\sigma_{n}(x)=\mathcal{N}\left(f_{n}(x), 1-u_{n}\right) \quad \text { for all } n \geq 0 \text { and } x \in S^{n}
$$

Such a $\sigma$ satisfies condition (ii) since $\sigma_{n+1}(x, y)$ depends only on the last observation $y$ and the mean of $\sigma_{n}(x)$. As shown in Section 4, $\sigma$ satisfies condition (i) as well. Moreover, under $P_{\sigma}$, the sequence $\left(X_{n}\right)$ is Gaussian with mean 0 , variance 1 , and a known covariance structure.

Due to its simple form, the above $\sigma$ is potentially useful in applications. In addition, $\sigma$ is just a special case of a larger class of strategies satisfying (i)-(ii). In fact, the normal distribution can be replaced by any symmetric stable law. For instance, the normal could be replaced by the Cauchy if heavier tails are regarded more suitable for prediction.

The last part of the paper is devoted to the asymptotics of $\sigma_{n}$ as $n \rightarrow \infty$. In fact, because of condition (i), one obtains

$$
P_{\sigma}\left(\sigma_{n} \rightarrow \mu \text { weakly }\right)=1
$$

for some random probability measure $\mu$ on $(S, \mathcal{B})$; see Subsection 2.2. Hence, it is quite natural to investigate $\mu$, and this is exactly the scope of Section 5. We give conditions for $\mu \ll \sigma_{0}$ a.s., for $\mu$ to be degenerate a.s., and for $\left\|\sigma_{n}-\mu\right\| \stackrel{a . s .}{\longrightarrow} 0$ where $\|\cdot\|$ is total variation norm. 
Finally, some applications are discussed in Section 6 .

To make the paper more readable, all the proofs are gathered in the "supplementary material".

\section{Preliminaries}

\subsection{Some further notation}

Let $\lambda, \nu \in \mathcal{P}$. We write $\lambda \ll \nu$ to mean that $\lambda$ is absolutely continuous with respect to $\nu$, namely, $\lambda(A)=0$ whenever $A \in \mathcal{B}$ and $\nu(A)=0$. Moreover, $\lambda$ and $\nu$ are singular if $\lambda(A)=\nu\left(A^{c}\right)=0$ for some $A \in \mathcal{B}$.

We denote by $x$ a point of $S^{n}$ where $n$ is an integer or $n=\infty$. In both cases, $x_{i}$ is the $i$-th coordinate of $x$. If $n=0$ and $\sigma$ is a strategy, $\sigma_{0}(x)$ is meant as $\sigma_{0}(x)=\sigma_{0}$. Moreover, if $x \in S^{\infty}$ and $f$ is any map on $S^{n}$, we write $f(x)$ to denote $f(x)=f\left(x_{1}, \ldots, x_{n}\right)$. In particular,

$$
\sigma_{n}(x):=\sigma_{n}\left(x_{1}, \ldots, x_{n}\right) \quad \text { for all } x \in S^{\infty}
$$

\subsection{Conditional identity in distribution}

C.i.d. sequences have been introduced in [4] and [22] and then investigated in various papers; see e.g. [1], [2], [5], [6], [7], [8], [9], [11], [15], [18], [19].

Let $P$ be a probability measure on $\left(S^{\infty}, \mathcal{B}^{\infty}\right)$. Say that $\left(X_{n}\right)$ is c.i.d. 
2.2 Conditional identity in distribution

(or that $P$ is c.i.d.) if $X_{2} \sim X_{1}$ and

$P\left(X_{k} \in \cdot \mid X_{1}, \ldots, X_{n}\right)=P\left(X_{n+1} \in \cdot \mid X_{1}, \ldots, X_{n}\right) \quad$ a.s. for all $k>n \geq 1$.

Thus, at each time $n$, the future observations $\left(X_{k}: k>n\right)$ are identically distributed given the past. This is actually weaker than exchangeability. Indeed, $\left(X_{n}\right)$ is exchangeable if and only if it is stationary and c.i.d.

The asymptotics of c.i.d. sequences is similar to that of exchangeable ones. To see this, suppose $P$ is c.i.d. and define the empirical measures

$$
\mu_{n}(x)=\frac{1}{n} \sum_{i=1}^{n} \delta_{x_{i}} \quad \text { for all } n \geq 1 \text { and } x \in S^{\infty}
$$

Define also

$$
\mu(x)=\lim _{n} \mu_{n}(x) \text { if the limit exists and } \mu(x)=\delta_{x_{1}} \text { otherwise, }
$$

where $x \in S^{\infty}$ and the limit is meant as a weak limit of probability measures.

The random probability measure $\mu$ is a meaningful parameter of $P$ (even if not as crucial as in the exchangeable case; see [8, Ex. 17]). In fact,

$$
\mu_{n}(A) \stackrel{\text { a.s. }}{\longrightarrow} \mu(A) \quad \text { for each } A \in \mathcal{B} \text {. }
$$

Moreover, for fixed $n \geq 0$ and $A \in \mathcal{B}$, one obtains

$$
E_{P}\left\{\mu(A) \mid X_{1}, \ldots, X_{n}\right\}=P\left(X_{n+1} \in A \mid X_{1}, \ldots, X_{n}\right) \text { a.s. }
$$

By martingale convergence, this equality implies

$$
P\left(X_{n+1} \in A \mid X_{1}, \ldots, X_{n}\right) \stackrel{\text { a.s. }}{\longrightarrow} \mu(A) \text { for each } A \in \mathcal{B} \text {. }
$$


We also note that $\left(X_{n}\right)$ is asymptotically exchangeable, in the sense that the probability distribution of the shifted sequence $\left(X_{n}, X_{n+1}, \ldots\right)$ converges weakly to an exchangeable probability measure $Q$ on $\left(S^{\infty}, \mathcal{B}^{\infty}\right)$. Furthermore, $Q=P$ on the $\sigma$-field generated by $\mu$.

Finally, we report from [6] a characterization of c.i.d. sequences in terms of strategies. The next result is fundamental for this paper.

Theorem 1. ([6, Th. 3.1]). For any strategy $\sigma,\left(X_{n}\right)$ is c.i.d. under $P_{\sigma}$ if and only if

$$
\sigma_{n}(x)(A)=\int \sigma_{n+1}(x, y)(A) \sigma_{n}(x)(d y)
$$

for all $n \geq 0$, all $A \in \mathcal{B}$ and $P_{\sigma}$-almost all $x \in S^{n}$.

Henceforth, we say " $P_{\sigma}$ is c.i.d." to mean that " $\left(X_{n}\right)$ is c.i.d. under $P_{\sigma}$ ".

\section{Convex combinations of random probability measures}

Let $\alpha=\left(\alpha_{0}, \alpha_{1}, \ldots\right)$ be a strategy and $q_{n}: S^{n} \rightarrow[0,1]$ a sequence of measurable functions, where $n \geq 0$ and $q_{0}$ is constant. For easy of notation, we write $\nu$ instead of $\alpha_{0}$, namely, we fix $\nu \in \mathcal{P}$ and we let $\alpha_{0}=\nu$. We also recall that $\alpha$ is Markovian if $\alpha_{n}(x)=\alpha_{n}^{*}\left(x_{n}\right)$ for all $n \geq 2$ and $x \in S^{n}$, where $\alpha_{n}^{*}: S \rightarrow \mathcal{P}$ is any measurable map. In this case, with a slight abuse of notation, we write $\alpha_{n}\left(x_{n}\right)$ instead of $\alpha_{n}^{*}\left(x_{n}\right)$. 
In this section, the strategy $\sigma$ satisfies equation (1.1), namely

$$
\sigma_{0}=\nu \quad \text { and } \quad \sigma_{n+1}(x, y)=q_{n}(x) \sigma_{n}(x)+\left(1-q_{n}(x)\right) \alpha_{n+1}(x, y)
$$

for all $n \geq 0, x \in S^{n}$ and $y \in S$. Arguing by induction, it follows that

$$
\sigma_{n}(x)=\nu \prod_{j=0}^{n-1} q_{j}+\sum_{i=1}^{n} \alpha_{i}\left(x_{1}, \ldots, x_{i}\right)\left(1-q_{i-1}\right) \prod_{j=i}^{n-1} q_{j}
$$

for all $n \geq 1$ and $x=\left(x_{1}, \ldots, x_{n}\right) \in S^{n}$. In formula $(3.3), \prod_{j=i}^{n-1} q_{j}$ is meant as 1 when $i=n$, and $q_{j}$ is a shorthand notation to denote

$$
q_{j}=q_{j}\left(x_{1}, \ldots, x_{j}\right)
$$

Our first goal is to give conditions for $P_{\sigma}$ to be c.i.d.

Theorem 2. $P_{\sigma}$ is c.i.d. provided

$$
\sigma_{n}(x)(A)=\int \alpha_{n+1}(x, y)(A) \sigma_{n}(x)(d y)
$$

for all $n \geq 0$, all $A \in \mathcal{B}$ and $P_{\sigma}$-almost all $x \in S^{n}$. Moreover, if $\alpha$ is Markovian, condition (3.4) follows from

$$
\alpha_{n}(x)(A)=\int \alpha_{n+1}(y)(A) \alpha_{n}(x)(d y)
$$

for all $n \geq 0$, all $A \in \mathcal{B}$ and $\nu$-almost all $x \in S$.

In the Markovian case, Theorem 2 applies if $\alpha_{n+1}$ is a conditional distribution of $\nu$ given $\mathcal{G}_{n}$ for all $n$, where $\left(\mathcal{G}_{n}\right)$ is any filtration on $(S, \mathcal{B})$. 
Corollary 1. Let $\mathcal{G}_{0} \subset \mathcal{G}_{1} \subset \mathcal{G}_{2} \subset \ldots \subset \mathcal{B}$ be an increasing sequence of sub- $\sigma$-fields of $\mathcal{B}$. If $\alpha$ is Markovian, then $P_{\sigma}$ is c.i.d. whenever

$$
\alpha_{n+1}(\cdot)(A)=E_{\nu}\left(1_{A} \mid \mathcal{G}_{n}\right), \quad \nu \text {-a.s., for all } n \geq 0 \text { and } A \in \mathcal{B}
$$

We are now able to provide examples of strategies which satisfy equation (1.1) and make $\left(X_{n}\right)$ c.i.d.

Example 1. (Copulas). A simple way to get the strategy $\alpha$ is to exploit an idea by Hahn, Martin and Walker [20]. To this end, we write "density" to mean "density with respect to Lebesgue measure". We also recall that, if $C$ is a bivariate copula and $F_{1}, F_{2}$ are distribution functions on $\mathbb{R}$, then $F(x, y)=C\left\{F_{1}(x), F_{2}(y)\right\}$ is a distribution function on $\mathbb{R}^{2}$. In addition, if $C, F_{1}$ and $F_{2}$ have densities, then

$$
f(x, y)=c\left\{F_{1}(x), F_{2}(y)\right\} f_{1}(x) f_{2}(y), \quad(x, y) \in \mathbb{R}^{2},
$$

is a density of $F$, where $c, f_{1}, f_{2}$ are densities of $C, F_{1}, F_{2}$, respectively.

Having noted this fact, let $S=\mathbb{R}$ and suppose that $\nu$ has a density $f_{0}$. Moreover, fix a sequence $C_{1}, C_{2}, \ldots$ of bivariate copulas with densities $c_{1}, c_{2}, \ldots$ For the sake of simplicity, assume $f_{0}>0$ and $c_{n}>0$ for all $n \geq 1$. Define $\sigma_{0}=\nu$ and denote by $F_{0}$ the distribution function corresponding to $\sigma_{0}$. Next, for each $x \in \mathbb{R}$, let

$$
\alpha_{1}(x)(d z)=f_{1}(z \mid x) d z \quad \text { where } \quad f_{1}(z \mid x)=c_{1}\left\{F_{0}(z), F_{0}(x)\right\} f_{0}(z) .
$$


Then, define $\sigma_{1}(x)=q_{0} \sigma_{0}+\left(1-q_{0}\right) \alpha_{1}(x)$ and call $F_{1}(\cdot \mid x)$ the distribution function corresponding to $\sigma_{1}(x)$. Next, for each $(x, y) \in \mathbb{R}^{2}$, let $\alpha_{2}(x, y)(d z)=f_{2}(z \mid x, y) d z$ where

$$
f_{2}(z \mid x, y)=c_{2}\left\{F_{1}(z \mid x), F_{1}(y \mid x)\right\} f_{1}(z \mid x)
$$

Then, define $\sigma_{2}(x, y)=q_{1}(x) \sigma_{1}(x)+\left(1-q_{1}(x)\right) \alpha_{2}(x, y)$. In general, suppose $\sigma_{n}(x)$ has been defined for all $x \in \mathbb{R}^{n}$ and denote by $f_{n}(\cdot \mid x)$ and $F_{n}(\cdot \mid x)$ the density and the distribution function of $\sigma_{n}(x)$. Then, it suffices to let

$$
\begin{aligned}
& \alpha_{n+1}(x, y)(d z)=f_{n+1}(z \mid x, y) d z \quad \text { for all } x \in \mathbb{R}^{n} \text { and } y \in \mathbb{R} \\
& \text { where } f_{n+1}(z \mid x, y)=c_{n+1}\left\{F_{n}(z \mid x), F_{n}(y \mid x)\right\} f_{n}(z \mid x) .
\end{aligned}
$$

Since $f_{n+1}(\cdot \mid x, y)$ is a density, $\alpha$ is a strategy dominated by Lebesgue measure. In addition, Fubini's theorem yields

$$
\begin{gathered}
\int \alpha_{n+1}(x, y)(A) \sigma_{n}(x)(d y)=\iint_{A} f_{n+1}(z \mid x, y) d z f_{n}(y \mid x) d y \\
=\int_{A} \int c_{n+1}\left\{F_{n}(z \mid x), F_{n}(y \mid x)\right\} f_{n}(z \mid x) f_{n}(y \mid x) d y d z \\
=\int_{A} f_{n}(z \mid x) d z=\sigma_{n}(x)(A) \quad \text { for all } A \in \mathcal{B} .
\end{gathered}
$$

Hence, $P_{\sigma}$ is c.i.d. because of Theorem 2 .

To implement Example 1, one only needs $f_{0}$ and the copula densities $c_{n}$. Some useful choices of $c_{n}$ are suggested in [20]. In particular, one can let $c_{n}=c_{1}$ for all $n$. Furthermore, one can use conditional copulas instead of 
plain copulas, in the sense that $c_{n+1}$ is allowed to depend on the observed data $x \in \mathbb{R}^{n}$. We also note that, letting $q_{n}=0$ for all $n$, the strategies obtained in [20] are a special case of Example 1.

In Example 1, the idea for building $\alpha$ is borrowed from [20]. A different idea is sketched in the next example.

Example 2. (Iterated conditioning). For each $\tau \in \mathcal{P}$ and each sub- $\sigma$ field $\mathcal{G} \subset \mathcal{B}$, let $\tau(\cdot \mid \mathcal{G})=\{\tau(\cdot \mid \mathcal{G})(x): x \in S\}$ denote a (regular) version of the conditional distribution of $\tau$ given $\mathcal{G}$. This means that $\tau(\cdot \mid \mathcal{G})(x)$ is a probability measure on $\mathcal{B}$, for fixed $x \in S$, and

$$
\tau(A \mid \mathcal{G})(\cdot)=E_{\tau}\left(1_{A} \mid \mathcal{G}\right), \quad \tau \text {-a.s., for all } A \in \mathcal{B}
$$

For each $n \geq 0$, take a sub- $\sigma$-field $\mathcal{G}_{n} \subset \mathcal{B}$. Define $\sigma_{0}=\nu$ and

$$
\alpha_{1}(x)=\nu\left(\cdot \mid \mathcal{G}_{0}\right)(x) \text { for all } x \in S
$$

To realize equation (1.1), define also $\sigma_{1}(x)=q_{0} \sigma_{0}+\left(1-q_{0}\right) \alpha_{1}(x)$. Next, for each $(x, y) \in S^{2}$, define

$\alpha_{2}(x, y)=\sigma_{1}(x)\left(\cdot \mid \mathcal{G}_{1}\right)(y) \quad$ and $\quad \sigma_{2}(x, y)=q_{1}(x) \sigma_{1}(x)+\left(1-q_{1}(x)\right) \alpha_{2}(x, y)$.

In general, after $\sigma_{n}(x)$ has been defined for all $x \in S^{n}$, it suffices to let

$$
\alpha_{n+1}(x, y)=\sigma_{n}(x)\left(\cdot \mid \mathcal{G}_{n}\right)(y) \quad \text { for all } x \in S^{n} \text { and } y \in S
$$

By construction, this strategy $\alpha$ satisfies condition (3.4). Hence, $P_{\sigma}$ is c.i.d. because of Theorem 2 . 
As an example, take $q_{n}=\frac{n+c}{n+1+c}$ and $\mathcal{G}_{n}=\mathcal{G}$ for all $n \geq 0$, where $c>0$ is a constant and $\mathcal{G} \subset \mathcal{B}$ a sub- $\sigma$-field. Then, formula (3.3) yields $\sigma_{n}(x)=\frac{c \nu+\sum_{i=1}^{n} \alpha_{i}\left(x_{1}, \ldots, x_{i}\right)}{n+c}=\frac{c \nu+\sum_{i=0}^{n-1} \sigma_{i}\left(x_{1}, \ldots, x_{i}\right)(\cdot \mid \mathcal{G})\left(x_{i+1}\right)}{n+c}$.

A special case of the latter strategy is discussed in Examples 4.5 .

If compared with Example 1, Example 2 replaces the choice of the copula densities $c_{n}$ with that of the sub- $\sigma$-fields $\mathcal{G}_{n}$. In principle, since the $\mathcal{G}_{n}$ are completely arbitrary, this provides some more degrees of freedom for modeling real situations. Obviously, however, the practical calculation of $\sigma_{n}(x)\left(\cdot \mid \mathcal{G}_{n}\right)(y)$ may be very hard.

We next turn to the Markovian case. In the rest of this Section, $\alpha$ is Markovian (i.e., $\alpha_{n}(x)=\alpha_{n}\left(x_{n}\right)$ for all $n \geq 2$ and $x \in S^{n}$ ).

Example 3. (Example 13 of [8]). For each $n \geq 0$, let $\mathcal{H}_{n}$ be a countable partition of $S$ such that $H \in \mathcal{B}$ and $\nu(H)>0$ for all $H \in \mathcal{H}_{n}$. Define

$$
\alpha_{n+1}(y)=\sum_{H \in \mathcal{H}_{n}} 1_{H}(y) \nu(\cdot \mid H)=\nu\left(\cdot \mid H_{y}^{n}\right) \quad \text { for all } y \in S
$$

where $H_{y}^{n}$ denotes the only $H \in \mathcal{H}_{n}$ such that $y \in H$. If $\mathcal{G}_{n}$ is the $\sigma$-field generated by $\mathcal{H}_{n}$, one obtains $\alpha_{n+1}(\cdot)(A)=E_{\nu}\left(1_{A} \mid \mathcal{G}_{n}\right)$ for all $A \in \mathcal{B}$. Moreover, $\mathcal{G}_{n} \subset \mathcal{G}_{n+1}$ provided $\mathcal{H}_{n+1}$ is finer than $\mathcal{H}_{n}$ for all $n \geq 0$ (as we assume). Therefore, $P_{\sigma}$ is c.i.d. because of Corollary 1.

Example 3 can be developed in various ways. For any partition $\mathcal{H}$ of 
$S$, let

$$
\mathcal{U}(\mathcal{H})=\sup _{H \in \mathcal{H}} \sup _{y, z \in H} d(y, z) \quad \text { where } d \text { is the distance on } S \text {. }
$$

Example 4. (Dirichlet-like sequences). Fix a constant $c>0$ and define

$$
q_{n}=\frac{n+c}{n+1+c}, \quad \alpha_{n+1}(y)=\nu\left(\cdot \mid H_{y}^{n}\right), \quad \nu_{n}(x)=\frac{\sum_{i=1}^{n} \nu\left(\cdot \mid H_{x_{i}}^{i-1}\right)}{n}
$$

Then, formula 3.3 yields

$$
\sigma_{n}(x)=\frac{c \nu+\sum_{i=1}^{n} \nu\left(\cdot \mid H_{x_{i}}^{i-1}\right)}{n+c}=\frac{c}{n+c} \nu+\frac{n}{n+c} \nu_{n}(x) .
$$

In turn, the predictives of a Dirichlet sequence are

$$
\beta_{n}(x)=\frac{c}{n+c} \nu+\frac{n}{n+c} \mu_{n}(x)
$$

where $\mu_{n}(x)=(1 / n) \sum_{i=1}^{n} \delta_{x_{i}}$ is the empirical measure. The strategies $\sigma$ and $\beta$ have a similar structure. Moreover, $\sigma_{n}(x)$ and $\beta_{n}(x)$ are usually close for large $n$. In fact, for various distances $D$ on $\mathcal{P}$, one obtains

$$
\lim _{n} D\left[\sigma_{n}(x), \beta_{n}(x)\right]=0 \quad \text { for each } x \in S^{\infty}
$$

provided $\lim _{n} \mathcal{U}\left(\mathcal{H}_{n}\right)=0$. For instance, relation $(3.6)$ holds if $D$ is the bounded Lipschitz metric; see Theorem 4. Despite (3.6), however, $\sigma$ and $\beta$ conflict under a fundamental aspect. Indeed, $\sigma_{n}(x) \ll \nu$ for all $n \geq 0$ and $x \in S^{n}$ while this is not true for $\beta_{n}(x)$. As a consequence, $P_{\sigma}$ and $P_{\beta}$ are even singular when $\nu$ is non-atomic; see Theorem 4 again. 
Example 5. (Example 4 continued). The situation of Example 4 may appear strange. Suppose $\nu$ is non-atomic and $\lim _{n} \mathcal{U}\left(\mathcal{H}_{n}\right)=0$. On one hand, since $P_{\sigma}$ and $P_{\beta}$ are singular, $\sigma$ and $\beta$ induce completely different distributions on the data. On the other hand, because of $(3.6), \sigma$ and $\beta$ provide similar predictions for large $n$.

Such a situation mostly depends on the distance $D$. In fact, $\sigma_{n}(x)$ and $\beta_{n}(x)$ are no longer close if $D$ is replaced by some stronger distance on $\mathcal{P}$, such as the total variation distance.

More precisely, suppose the target is to predict $f\left(X_{n+1}\right)$ based on $\left(X_{1}, \ldots, X_{n}\right)$, where $f: S \rightarrow \mathbb{R}$ is a bounded measurable function. Then, $\sigma$ and $\beta$ actually yield similar predictions for large $n$. As an example, if $f$ is Lipschitz and $D$ is the bounded Lipschitz metric, one obtains

$$
\begin{gathered}
\left|E_{\sigma}\left\{f\left(X_{n+1}\right) \mid\left(X_{1}, \ldots, X_{n}\right)=x\right\}-E_{\beta}\left\{f\left(X_{n+1}\right) \mid\left(X_{1}, \ldots, X_{n}\right)=x\right\}\right| \\
=\left|\int f(t) \sigma_{n}(x)(d t)-\int f(t) \beta_{n}(x)(d t)\right| \leq k D\left[\sigma_{n}(x), \beta_{n}(x)\right]
\end{gathered}
$$

for some constant $k$ depending only on $f$.

However, $\sigma$ and $\beta$ give conflicting predictions in more elaborated problems. For instance, suppose one aims to predict whether the next observation is new. Letting $G_{n}=\left\{X_{n+1}=X_{i}\right.$ for some $\left.i \leq n\right\}$, one obtains

$$
\begin{gathered}
P_{\sigma}\left(G_{n} \mid\left(X_{1}, \ldots, X_{n}\right)=x\right)=\sigma_{n}(x)\left(\left\{x_{1}, \ldots, x_{n}\right\}\right)=0 \quad \text { while } \\
P_{\beta}\left(G_{n} \mid\left(X_{1}, \ldots, X_{n}\right)=x\right)=\beta_{n}(x)\left(\left\{x_{1}, \ldots, x_{n}\right\}\right)=n /(n+c) .
\end{gathered}
$$


Example 6. (Exponential smoothing-like sequences). Let

$$
\beta_{n}(x)=q^{n} \nu+(1-q) \sum_{i=1}^{n} q^{n-i} \delta_{x_{i}}
$$

where $q \in[0,1]$ is any constant. Making predictions through $\beta$ may be reasonable when the forecaster has only vague opinions on the dependence structure of the data, and yet she feels that the weight of the $i$-th observation $x_{i}$ should be an increasing function of $i$; see [2] and [8]. Now, if $q_{n}=q$ and $\alpha_{n+1}(y)=\nu\left(\cdot \mid H_{y}^{n}\right)$, formula 3.3 reduces to

$$
\sigma_{n}(x)=q^{n} \nu+(1-q) \sum_{i=1}^{n} q^{n-i} \nu\left(\cdot \mid H_{x_{i}}^{i-1}\right) .
$$

Essentially the same remarks of Examples 445, about the connections between $\sigma$ and $\beta$, can be repeated in this example.

Exploiting countable partitions is a flexible idea which may be realized in various ways. We support this claim with two further examples.

Example 7. (Mixed strategies). Let $\mathcal{H} \subset \mathcal{B}$ be a fixed countable partition of $S$ and $A_{0} \subset A_{1} \subset A_{2} \subset \ldots$ an increasing sequence of elements of $\mathcal{B}$. Assume $\nu\left(A_{n}^{c} \cap H\right)>0$ whenever $A_{n}^{c} \cap H \neq \emptyset$ and define

$$
\alpha_{n+1}(y)=1_{A_{n}}(y) \delta_{y}+1_{A_{n}^{c}}(y) \nu\left(\cdot \mid A_{n}^{c} \cap H_{y}\right)
$$

where $H_{y}$ is the only $H \in \mathcal{H}$ such that $y \in H$. Then, $\alpha_{n+1}$ satisfies Corollary 1 with $\mathcal{G}_{n}$ the $\sigma$-field generated by the sets $A \cap A_{n}$ and $H \cap A_{n}^{c}$ for all $A \in \mathcal{B}$ and $H \in \mathcal{H}$. Since $\mathcal{G}_{n} \subset \mathcal{G}_{n+1}$ for all $n$, it follows that $P_{\sigma}$ is c.i.d. 
For instance, take

$$
S=\mathbb{R}, \quad \mathcal{H}=\{(-\infty, 0),\{0\},(0, \infty)\}, \quad A_{n}=\left[-u_{n}, u_{n}\right]
$$

where $0<u_{0}<u_{1}<u_{2}<\ldots$ are any constants. Suppose also that, at each time $n$, an observation $y$ is informative about the future observations whenever $|y| \leq u_{n}$. Otherwise, if $|y|>u_{n}$, the only relevant information provided by $y$ is its sign. Then, choosing $\alpha_{n+1}$ as above may be reasonable. Finally, taking $q_{n}$ as in Example 4, one obtains

$$
\begin{gathered}
\sigma_{n}(x)=\frac{c \nu+\sum_{i=1}^{n} \alpha_{i}\left(x_{i}\right)}{n+c} \\
=\frac{c \nu+\sum_{i=0}^{n-1}\left\{1_{A_{i}}\left(x_{i+1}\right) \delta_{x_{i+1}}+1_{B_{i}}\left(x_{i+1}\right) \nu\left(\cdot \mid B_{i}\right)+1_{C_{i}}\left(x_{i+1}\right) \nu\left(\cdot \mid C_{i}\right)\right\}}{n+c}
\end{gathered}
$$

where $B_{i}=\left(-\infty,-u_{i}\right)$ and $C_{i}=\left(u_{i}, \infty\right)$.

Example 8. (Occupancy models). At each time $n$, a random integer $r_{n}$ is selected and $r_{n}$ particles are randomly placed into $p$ boxes. The $i$-th observation is $x_{i}=\left(j_{1}(i), \ldots, j_{p}(i)\right)$ where $j_{k}(i)$ is the number of particles in box $k$ at time $i$. To model this situation, set $S=\{0,1,2, \ldots\}^{p}$ and take $\mathcal{H}_{n}=\mathcal{F}$ for all $n$, where $\mathcal{F}$ is the partition of $S$ with elements

$$
F_{r}=\left\{\left(j_{1}, \ldots, j_{p}\right) \in S: \sum_{k=1}^{p} j_{k}=r\right\} \quad \text { for } r=0,1,2, \ldots
$$

Moreover, take $\nu$ to be the probability distribution of $\left(Y_{1}, \ldots, Y_{p}\right)$ where $Y_{1}, \ldots, Y_{p}$ are i.i.d. Poisson random variables. The conditional distribution 
of $\left(Y_{1}, \ldots, Y_{p}\right)$ given $\sum_{k=1}^{p} Y_{k}=r$ is multinomial with index $r$ and equal cell probabilities $1 / p$. Therefore,

$$
\nu\left(\left\{\left(j_{1}, \ldots, j_{p}\right)\right\} \mid F_{r}\right)=\frac{1}{p^{r}} \frac{r !}{j_{1} ! \ldots j_{p} !} \text { for all }\left(j_{1}, \ldots, j_{p}\right) \in F_{r} .
$$

Hence, denoting by $x_{i}^{*}=\sum_{k=1}^{p} j_{k}(i)$ the sum of the coordinates of $x_{i}$, the strategy $\sigma$ is

$$
\sigma_{n}(x)=\nu \prod_{j=0}^{n-1} q_{j}+\sum_{i=1}^{n} \nu\left(\cdot \mid F_{x_{i}^{*}}\right)\left(1-q_{i-1}\right) \prod_{j=i}^{n-1} q_{j}
$$

The choice of $q_{j}$ depends on the specific problem at issue. For instance, $q_{j}$ could be as in Examples 4, 6 or 9. We just note that exchangeability is useful in the framework of occupancy models, and $P_{\sigma}$ is actually exchangeable (and not only c.i.d.) if $q_{j}=(j+c) /(j+1+c)$; see [10] and [13].

Our last example deals with a more elaborate choice of $q_{n}$.

Example 9. (Reinforcements). For each $n \geq 1$, fix a set $C_{n} \in \mathcal{B}^{n}$, two constants $0<a_{n}<1 / 2<b_{n}<1$, and define

$$
q_{n}(x)=b_{n} 1_{C_{n}}(x)+a_{n}\left(1-1_{C_{n}}(x)\right) \quad \text { for all } x \in S^{n} .
$$

Roughly speaking, the underlying idea is that $\sigma_{n}(x)$ exhibits good predictive performances whenever $x \in C_{n}$. Therefore, if $\left(X_{1}, \ldots, X_{n+1}\right)=(x, y)$ and $x \in C_{n}$, to predict $X_{n+2}$ the forecaster is inclined to reinforce $\sigma_{n}(x)$ with respect to $\alpha_{n+1}(y)$. (Recall that $\left.a_{n}<1 / 2<b_{n}\right)$. 
As a concrete example, let $S=[0,1]$ and $q_{0}=1 / 2$. For all $n \geq 1$ and $x \in S^{n}$, let $\bar{x}_{n}=(1 / n) \sum_{i=1}^{n} x_{i}$ be the sample mean of $x$ and $m_{n}(x)$ any (measurable) predictor of $X_{n+1}$ based on $\sigma_{n}(x)$. For definiteness,

$$
m_{n}(x)=\int t \sigma_{n}(x)(d t)
$$

If $m_{n}(x)$ is regarded as a predictor of the past observations $x_{i}, i \leq n$, then

$$
\bar{x}_{n}-m_{n}(x)=(1 / n) \sum_{i=1}^{n}\left\{x_{i}-m_{n}(x)\right\}
$$

is the arithmetic mean of the prediction errors. In a sense, $\sigma_{n}(x)$ works nicely whenever $\bar{x}_{n}-m_{n}(x)$ is small. Hence, given $\epsilon>0$, one could let

$$
C_{n}=\left\{x \in S^{n}:\left|\bar{x}_{n}-m_{n}(x)\right|<\epsilon\right\}
$$

To close this section, we note that the strategies obtained so far have applications beyond the predictive framework of this paper. In fact, various species sampling sequences correspond to strategies of the form (3.3). And, in Bayesian nonparametrics, species sampling sequences may be used to define priors; see [1].

\section{Predictions via stable laws}

In this section, we let $S=\mathbb{R}$, we fix a constant $\gamma \in(0,2]$, and we introduce a further class of strategies. Such strategies need not satisfy equation (1.1) (unless $q_{n}=0$ for all $n$ ). However, they meet conditions (i)-(ii) and the 
probability measure $\sigma_{n}(x)$ is $\gamma$-stable for all $n \geq 0$ and $x \in S^{n}$. (The exponent $\gamma$ of a stable law is usually denoted by $\alpha$, but in this paper $\alpha$ denotes a strategy).

Let $Z$ be a real random variable with characteristic function

$$
E\{\exp (i t Z)\}=\exp \left(-\frac{|t|^{\gamma}}{2}\right) \quad \text { for all } t \in \mathbb{R} \text {. }
$$

For $a \in \mathbb{R}$ and $b>0$, denote by $\mathcal{S}(a, b)$ the probability distribution of $a+b^{1 / \gamma} Z$, namely

$$
\mathcal{S}(a, b)(A)=P\left(a+b^{1 / \gamma} Z \in A\right) \quad \text { for all } A \in \mathcal{B} \text {. }
$$

Note that $\mathcal{S}(a, b)=\mathcal{N}(a, b)$ if $\gamma=2$, where $\mathcal{N}(a, b)$ is the Gaussian law on $\mathcal{B}$ with mean $a$ and variance $b$. Similarly, $\mathcal{S}(a, b)=\mathcal{C}(a, b)$ if $\gamma=1$, where $\mathcal{C}(a, b)$ is the probability measure on $\mathcal{B}$ with density $f(x)=\frac{2 b}{\pi} \frac{1}{b^{2}+4(x-a)^{2}}$. (Incidentally, in this parametrization, the standard Cauchy distribution is $\mathcal{C}(0,2)$ and not $\mathcal{C}(0,1))$

Next, fix the real numbers

$$
0=u_{0}<u_{1}<u_{2}<\ldots<u
$$

and define $f_{0}=0$ and

$$
f_{n+1}(x, y)=f_{n}(x)\left(1-\left(\frac{u_{n+1}-u_{n}}{u-u_{n}}\right)^{1 / \gamma}\right)+y\left(\frac{u_{n+1}-u_{n}}{u-u_{n}}\right)^{1 / \gamma}
$$

for all $n \geq 0, x \in S^{n}$ and $y \in S$. 
In this section, we focus on the strategy

$$
\sigma_{n}(x)=\mathcal{S}\left(f_{n}(x), u-u_{n}\right) \quad \text { for all } n \geq 0 \text { and } x \in S^{n} .
$$

It is worth noting that $\sigma_{0}=\mathcal{S}(0, u)$ and $\sigma_{n+1}(x, y)$ can be easily evaluated based on $y$ and the median of $\sigma_{n}(x)$. Hence, condition (ii) holds. We now turn to condition (i).

Theorem 3. If $\sigma$ is given by (4.7), then $P_{\sigma}$ is c.i.d.

In the rest of this section, $\sigma$ denotes the strategy (4.7).

An useful feature of $\sigma$ is its asymptotic behavior. Define

$$
L=\left\{x \in S^{\infty}: \lim _{n} f_{n}(x) \text { exists and is finite }\right\}
$$

and $f(x)=\lim _{n} f_{n}(x)$ for each $x \in L$. Since $P_{\sigma}$ is c.i.d., it follows that $P_{\sigma}(L)=1$. Moreover, for each $x \in L$, one obtains

$$
\begin{gathered}
\sigma_{n}(x) \longrightarrow \delta_{f(x)} \text { weakly if } \sup _{n} u_{n}=u \text { and } \\
\sigma_{n}(x) \longrightarrow \mathcal{S}\left(f(x), u-\sup _{n} u_{n}\right) \text { in total variation if } \sup _{n} u_{n}<u .
\end{gathered}
$$

We refer to the proof of Theorem 6 for more details. Here, we turn to examples.

Example 10. (Cauchy and Normal distributions). The most popular cases are $\gamma=1$ and $\gamma=2$. Indeed,

$$
\sigma_{n}(x)=\mathcal{C}\left(f_{n}(x), u-u_{n}\right) \quad \text { or } \quad \sigma_{n}(x)=\mathcal{N}\left(f_{n}(x), u-u_{n}\right)
$$


according to whether $\gamma=1$ or $\gamma=2$. Both strategies can be useful in real problems. Note also that $f_{n}(x)$ is just a weighted average of the first $n$ observations $x_{1}, \ldots, x_{n}$ and, in the normal case, the weights are connected to the conditional variances.

The next example provides further information on the sequence $\left(X_{n}\right)$.

Example 11. (Finite dimensional distributions). Let

$$
Y_{n+1}=\sum_{i=1}^{n}\left(u_{i}-u_{i-1}\right)^{1 / \gamma} Z_{i}+\left(u-u_{n}\right)^{1 / \gamma} Z_{n+1} \quad \text { for all } n \geq 0
$$

where $Z_{1}, Z_{2}, \ldots$ is an i.i.d. sequence with $Z_{1} \sim \mathcal{S}(0,1)$. Then, $Y_{1} \sim \mathcal{S}(0, u)$. Furthermore,

$$
\left(Y_{1}, \ldots, Y_{n}\right)=g_{n}\left(Z_{1}, \ldots, Z_{n}\right) \text { and } \sum_{i=1}^{n}\left(u_{i}-u_{i-1}\right)^{1 / \gamma} Z_{i}=f_{n}\left(Y_{1}, \ldots, Y_{n}\right)
$$

where $g_{n}$ is an invertible linear transformation. Therefore,

$$
\begin{aligned}
& P\left(Y_{n+1} \in \cdot \mid Y_{1}, \ldots, Y_{n}\right)=P\left(Y_{n+1} \in \cdot \mid Z_{1}, \ldots, Z_{n}\right) \\
= & P\left(f_{n}\left(Y_{1}, \ldots, Y_{n}\right)+\left(u-u_{n}\right)^{1 / \gamma} Z_{n+1} \in \cdot \mid Z_{1}, \ldots, Z_{n}\right) \\
= & \mathcal{S}\left(f_{n}\left(Y_{1}, \ldots, Y_{n}\right), u-u_{n}\right)=\sigma_{n}\left(Y_{1}, \ldots, Y_{n}\right) \quad \text { a.s. }
\end{aligned}
$$

In other terms, the predictive distributions of the sequence $\left(Y_{n}\right)$ agree with those of $\sigma$, and this implies

$$
P_{\sigma}(B)=P\left(\left(Y_{1}, Y_{2}, \ldots\right) \in B\right) \quad \text { for all } B \in \mathcal{B}^{\infty} \text {. }
$$


This equation allows to determine the finite dimensional distributions of $\left(X_{n}\right)$ under $P_{\sigma}$. Here, we just highlight two facts. Firstly,

$$
f_{n}\left(Y_{1}, \ldots, Y_{n}\right)=\sum_{i=1}^{n}\left(u_{i}-u_{i-1}\right)^{1 / \gamma} Z_{i} \sim u_{n}^{1 / \gamma} Z_{1} \sim \mathcal{S}\left(0, u_{n}\right)
$$

Thus, $f_{n} \sim \mathcal{S}\left(0, u_{n}\right)$ under $P_{\sigma}$, namely, $P_{\sigma}\left(f_{n} \in A\right)=\mathcal{S}\left(0, u_{n}\right)(A)$ for all $A \in \mathcal{B}$. Secondly, since $g_{n}$ is linear, the finite dimensional distributions of $\left(X_{n}\right)$ under $P_{\sigma}$ are Gaussian when $\gamma=2$. In this case, since $\left(Y_{n}\right)$ is c.i.d., the moments are

$$
\begin{gathered}
E_{P_{\sigma}}\left(X_{n}\right)=0, \quad E_{P_{\sigma}}\left(X_{n}^{2}\right)=u \quad \text { and } \\
E_{P_{\sigma}}\left(X_{n} X_{m}\right)=E\left(Y_{n} Y_{m}\right)=E\left[Y_{n} E\left(Y_{m} \mid Y_{1}, \ldots, Y_{n}\right)\right] \\
=E\left(Y_{n} Y_{n+1}\right)=u_{n-1}+\sqrt{\left(u_{n}-u_{n-1}\right)\left(u-u_{n-1}\right)} \quad \text { for all } 1 \leq n<m .
\end{gathered}
$$

The last example collects some miscellaneous remarks.

Example 12. (Choice of $\gamma, u$ and $u_{n}$ ). To work with $\sigma$, one has only to select $\gamma$ and $u, u_{1}, u_{2}, \ldots$ Obviously, the choice of $\gamma$ depends on the specific problem at hand. We just note that, in applications, $\gamma \in\{1,2\}$ is not the unique meaningful choice. For instance, $\gamma \notin\{1,2\}$ is quite common when modeling financial data; see e.g. [24, Chap. 13]. The numbers $u$ and $u_{n}$ are scale parameters which control the dispersion structure of $\left(X_{n}\right)$. If $\gamma=2$, for instance, $u$ and $u_{n}$ determine the variances and covariances of the Gaussian sequence $\left(X_{n}\right)$; see Example 11. An important distinction is $\sup _{n} u_{n}=u$ or 
$\sup _{n} u_{n}<u$, as the limiting distribution of $\sigma_{n}$ is degenerate in the former case while it is not in the latter. Finally, we mention a practically useful choice of $u_{n}$. Fix $u>0$ and $q \in(0,1)$ and define

$$
u_{n}=u\left(1-q^{n}\right) \quad \text { for all } n \geq 0 .
$$

Then, $u_{n+1}-u_{n}=\left(u-u_{n}\right)(1-q)$ and the updating rule for $f_{n}$ reduces to

$$
f_{n+1}(x, y)=(1-b) f_{n}(x)+b y \quad \text { where } b=(1-q)^{1 / \gamma} \text {. }
$$

Equivalently, $f_{n}(x)=b \sum_{j=1}^{n}(1-b)^{n-j} x_{j}$ for each $x \in S^{n}$.

\section{Asymptotics}

We first recall two popular distances on $\mathcal{P}$. Let $\lambda_{1}, \lambda_{2} \in \mathcal{P}$ and let $F$ be the set of all functions $f: S \rightarrow[-1,1]$ such that $|f(y)-f(z)| \leq d(y, z)$ for all $y, z \in S$, where $d$ is the distance on $S$. The bounded Lipschitz metric and the total variation distance are, respectively,

$$
D\left(\lambda_{1}, \lambda_{2}\right)=\sup _{f \in F}\left|\int f d \lambda_{1}-\int f d \lambda_{2}\right| \quad \text { and } \quad\left\|\lambda_{1}-\lambda_{2}\right\|=\sup _{A \in \mathcal{B}}\left|\lambda_{1}(A)-\lambda_{2}(A)\right|
$$

It is not hard to see that $D \leq 2\|\cdot\|$. Moreover, $D$ metrizes weak convergence of probability measures, in the sense that, for all $\lambda_{n}, \lambda \in \mathcal{P}$,

$$
\lambda_{n} \rightarrow \lambda \text { weakly } \Leftrightarrow \lim _{n} D\left(\lambda_{n}, \lambda\right)=0
$$

We next make precise some claims made in Example 4. 
Theorem 4. Let $\sigma$ and $\beta$ be as in Example 4. If $\lim _{n} \mathcal{U}\left(\mathcal{H}_{n}\right)=0$, then

$$
\lim _{n} D\left[\sigma_{n}(x), \beta_{n}(x)\right]=0 \quad \text { for each } x \in S^{\infty} .
$$

Moreover, $P_{\sigma}$ and $P_{\beta}$ are singular if $\nu$ is non-atomic.

Next, for each $x \in S^{\infty}$, define

$$
\mu(x)=\lim _{n} \mu_{n}(x) \text { if the limit exists and } \mu(x)=\delta_{x_{1}} \text { otherwise, }
$$

where $\mu_{n}(x)=(1 / n) \sum_{i=1}^{n} \delta_{x_{i}}$ is the empirical measure and the limit is meant as a weak limit of probability measures. The random probability measure $\mu$ is a meaningful object. In fact,

$$
P_{\sigma}\left\{x \in S^{\infty}: \sigma_{n}(x) \rightarrow \mu(x) \text { weakly }\right\}=1
$$

for any strategy $\sigma$ such that $P_{\sigma}$ is c.i.d.; see Subsection 2.2. In the sequel, we investigate $\mu$ when $\sigma$ comes from Sections 34.4 .

For each $\tau \in \mathcal{P}$, say that $\tau$ is degenerate if $\tau=\delta_{z}$ for some $z \in S$. The abbreviation "a.s." stands for "P $P_{\sigma}$-a.s." For instance, $\mu \ll \tau$ a.s. means $\mu(x) \ll \tau$ for $P_{\sigma}$-almost all $x \in S^{\infty}$. Recall also that $q_{n}(x)=q_{n}\left(x_{1}, \ldots, x_{n}\right)$ for all $x \in S^{\infty}$.

Theorem 5. If the strategy $\sigma$ satisfies equation (1.1), then $\sigma_{n}(x)$ converges in total variation distance for each $x \in S^{\infty}$ such that $\sum_{n}\left(1-q_{n}(x)\right)<\infty$. Moreover, if $\sigma$ is as in Example 3, then: 
- $\mu \ll \nu$ a.s. and $\lim _{n}\left\|\sigma_{n}-\mu\right\|=0$ a.s. provided $\sum_{n}\left(1-q_{n}\right)<\infty$ a.s.;

- $\mu$ is degenerate a.s. provided $\lim _{n} \mathcal{U}\left(\mathcal{H}_{n}\right)=0$ and there are constants $a>0$ and $c_{n} \geq 0$ such that

$$
\sum_{n} c_{n}^{2}=\infty \quad \text { and } \quad a \leq q_{n} \leq 1-c_{n} \text { a.s. for all } n \geq 0 \text {. }
$$

Theorem 5 can be applied to the examples of Section 3. Suppose in fact $\lim _{n} \mathcal{U}\left(\mathcal{H}_{n}\right)=0$. Then, in Example 6, $\mu$ is degenerate a.s. In Example 9 $\mu \ll \nu$ a.s. if $\sum_{n}\left(1-b_{n}\right)<\infty$ and $\mu$ is degenerate a.s. if $\sum_{n}\left(1-b_{n}\right)^{2}=\infty$ and $\inf _{n} a_{n}>0$. However, Theorem 5 does not work in Example 4, for in that case $1-q_{n}(x)=1 /(n+1+c)$ for all $x \in S^{\infty}$. Indeed, the behavior of $\mu$ in Example 4 is an open problem.

Finally, we turn to the strategies of Section 4.

Theorem 6. In the notation of Section 4, let

$$
\begin{gathered}
L=\left\{x \in S^{\infty}: \lim _{n} f_{n}(x) \text { exists and is finite }\right\} \\
f(x)=\lim _{n} f_{n}(x) \text { for each } x \in L \quad \text { and } \quad u^{*}=\sup _{n} u_{n} .
\end{gathered}
$$

If $\sigma$ is the strategy (4.7) then, for each $x \in L$,

$$
\begin{gathered}
\sigma_{n}(x) \longrightarrow \delta_{f(x)} \text { weakly if } u^{*}=u \text { and } \\
\sigma_{n}(x) \longrightarrow \mathcal{S}\left(f(x), u-u^{*}\right) \text { in total variation if } u^{*}<u .
\end{gathered}
$$

Moreover, $P_{\sigma}(L)=1$ and $f \sim \mathcal{S}\left(0, u^{*}\right)$ under $P_{\sigma}$, namely

$$
P_{\sigma}(f \in A)=\mathcal{S}\left(0, u^{*}\right)(A) \quad \text { for all } A \in \mathcal{B} \text {. }
$$




\section{Applications}

We finally discuss some applications of the strategies obtained via NSA. We let $S=\mathbb{R}$ and we denote by $x \in \mathbb{R}^{n}$ the observed data.

Roughly speaking, NSA replaces the choice of the prior with that of a strategy $\sigma$; see Section 1. Thus, in general, NSA applies to any Bayesian prediction problem. Practically, for any time series $\left(X_{n}\right)$, the forecaster has only to choose her strategy $\sigma$. In making this choice, she has no constraints other than her feelings and the specific features of $\left(X_{n}\right)$. Once $\sigma$ is selected, its possible uses are the usual ones. For instance, the forecaster can build a pointwise predictor for $X_{n+1}$, such as the mean or the median of $\sigma_{n}(x)$. Or else, given $\gamma \in(0,1)$, she can build a prediction interval for $X_{n+1}$, namely an interval $I_{n}(x)$ such that

$$
P_{\sigma}\left(X_{n+1} \in I_{n}(x) \mid\left(X_{1}, \ldots, X_{n}\right)=x\right)=\sigma_{n}(x)\left[I_{n}(x)\right] \geq 1-\gamma
$$

The previous remarks, while reasonable, may look generic. Thus, we mention a more concrete application based on martingale posterior distributions (m.p.d.'s) as defined in [15]. A m.p.d. is the conditional distribution of $\theta$ given the observed data, where $\theta=\theta\left(X_{1}, X_{2}, \ldots\right)$ is any (measurable) function of the whole data sequence $\left(X_{1}, X_{2}, \ldots\right)$. Note that $\theta$ would be known if we knew $\left(X_{1}, X_{2}, \ldots\right)$. Hence, the only source of uncertainty is the ignorance about $\left(X_{n+1}, X_{n+2}, \ldots\right)$. Quoting from [15, Abst.], a m.p.d. 
"returns Bayesian uncertainty directly on any statistic of interest without the need for the likelihood and prior".

In applications, m.p.d.'s can be sampled through a computational scheme, called predictive resampling and displayed in Algorithm 1 below. Based on predictive resampling, in [15], several applications to real datasets are provided, including the galaxy and air quality datasets which are classic benchmarks to test new procedures.

Algorithm $1 \mathrm{~A}$ practical algorithm for predictive resampling

Assign $\sigma_{n}(x)$ based on the observed data $x=\left(x_{1}, \ldots, x_{n}\right)$

Set $\sigma_{n}^{*}(x)=\sigma_{n}(x)$

$M$ and $N>n$ are integers with $N$ large

for $j \leftarrow 1$ to $M$ do

for $i \leftarrow n+1$ to $N$ do

Sample $Y_{i} \sim \sigma_{i-1}^{*}$ where $\sigma_{i-1}^{*}=\sigma_{i-1}^{*}\left(x, Y_{n+1}, \ldots, Y_{i-1}\right)$

Update $\sigma_{i}^{*} \leftarrow\left\{\sigma_{i-1}^{*}, Y_{i}\right\}$

\section{end for}

Compute the empirical measure $\mu_{N}=\frac{1}{N}\left(\sum_{i=1}^{n} \delta_{x_{i}}+\sum_{i=n+1}^{N} \delta_{Y_{i}}\right)$ Compute $\theta_{N}^{(j)}$ according to $\mu_{N}$.

end for

Return $\theta_{N}^{(1)}, \ldots, \theta_{N}^{(M)}$ where the $\theta_{N}^{(j)}$ are estimates of $\theta$ based on $\mu_{N}$

M.p.d.'s are introduced in the framework of NSA. As in this paper, the 


\section{REFERENCES}

predictives are assigned directly and $\left(X_{n}\right)$ is required to be c.i.d. Condition

(ii) is very useful as well. Therefore, each of the strategies of Sections 3,4 can be exploited to obtain m.p.d.'s. To implement Algorithm 1, in fact, one needs to sample from a given predictive distribution. In turn, sampling from the strategies of Sections 3,4 is straightforward. In this sense, using such strategies in predictive resampling is computationally efficient.

Acknowledgments: We are grateful to the editors and an anonymous referee for their useful remarks and suggestions.

\section{References}

[1] Airoldi E.M., Costa T., Bassetti F., Leisen F., Guindani M. (2014) Generalized species sampling priors with latent beta reinforcements, J.A.S.A., 109, 1466-1480.

[2] Bassetti F., Crimaldi I., Leisen F. (2010) Conditionally identically distributed species sampling sequences, Adv. Appl. Probab., 42, 433-459.

[3] Berti P., Regazzini E., Rigo P. (1997) Well-calibrated, coherent forecasting systems, Theory Probab. Appl., 42, 82-102.

[4] Berti P., Pratelli L., Rigo P. (2004) Limit theorems for a class of identically distributed random variables, Ann. Probab., 32, 2029-2052. 


\section{REFERENCES}

[5] Berti P., Crimaldi I., Pratelli L., Rigo P. (2009) Rate of convergence of predictive distributions for dependent data, Bernoulli, 15, 1351-1367.

[6] Berti P., Pratelli L., Rigo P. (2012) Limit theorems for empirical processes based on dependent data, Electronic J. Probab., 17, 1-18.

[7] Berti P., Pratelli L., Rigo P. (2013) Exchangeable sequences driven by an absolutely continuous random measure, Ann. Probab., 41, 20902102.

[8] Berti P., Dreassi E., Pratelli L., Rigo P. (2021) A class of models for Bayesian predictive inference, Bernoulli, 27, 702-726.

[9] Berti P., Dreassi E., Pratelli L., Rigo P. (2021) Asymptotics of certain conditionally identically distributed sequences, Statist. Prob. Lett., $168,1-10$.

[10] Berti P., Dreassi E., Leisen F., Pratelli L., Rigo P. (2021) Kernel based Dirichlet sequences, arXiv:2106.00114 [math.PR].

[11] Cassese A., Zhu W., Guindani M., Vannucci M. (2019) A Bayesian nonparametric spiked process prior for dynamic model selection, Bayesian Analysis, 14, 553-572.

[12] Cifarelli D.M., Regazzini E. (1996) De Finetti's contribution to probability and statistics, Statist. Science, 11, 253-282. 


\section{REFERENCES}

[13] Collet F., Leisen F., Spizzichino F., Suter F. (2013) Exchangeable occupancy models and discrete processes with the generalized uniform order statistics property, Probability in the Engineering and Informational Sciences, 27, 533-552.

[14] Dubins L.E., Savage L.J. (1965) How to gamble if you must: Inequalities for stochastic processes, McGraw Hill.

[15] Fong E., Holmes C., Walker S.G. (2021) Martingale posterior distributions, arXiv:2103.15671v1

[16] Fortini S., Ladelli L., Regazzini E. (2000) Exchangeability, predictive distributions and parametric models, Sankhya A, 62, 86-109.

[17] Fortini S., Petrone S. (2012) Predictive construction of priors in Bayesian nonparametrics, Brazilian J. Probab. Statist., 26, 423-449.

[18] Fortini S., Petrone S., Sporysheva P. (2018) On a notion of partially conditionally identically distributed sequences, Stoch. Proc. Appl., 128, 819-846.

[19] Fortini S., Petrone S. (2020) Quasi-Bayes properties of a procedure for sequential learning in mixture models, J. Royal Stat. Soc. B, 82, $1087-1114$. 


\section{REFERENCES}

[20] Hahn P.R., Martin R., Walker S.G. (2018) On recursive Bayesian predictive distributions, J.A.S.A., 113, 1085-1093.

[21] Hill B.M. (1993) Parametric models for $A_{n}$ : splitting processes and mixtures, J. Royal Stat. Soc. B, 55, 423-433.

[22] Kallenberg O. (1988) Spreading and predictable sampling in exchangeable sequences and processes, Ann. Probab., 16, 508-534.

[23] Lee J., Quintana F.A., Muller P., Trippa L. (2013) Defining predictive probability functions for species sampling models, Statist. Science, 28, 209-222.

[24] Mc Culloch J.H. (1996) Financial applications of stable distributions, in: Statistical methods in finance (Maddala G.S. and Rao C.R. Eds.), North Holland, Elsevier Science BV, Amsterdam.

[25] Pitman J. (1996) Some developments of the Blackwell-MacQueen urn scheme, Statistics, Probability and Game Theory, IMS Lect. Notes Mon. Series, 30, 245-267.

[26] Pitman J. (2006) Combinatorial stochastic processes, Lectures from the XXXII Summer School in Saint-Flour, 2002, Springer, Berlin.

${ }^{1}$ Dipartimento di Scienze Fisiche, Informatiche e Matematiche, Università di Modena e Reggio-Emilia, via Campi 213/B, 41100 Modena, Italy 


\section{REFERENCES}

E-mail: patrizia.berti@unimore.it

${ }^{2}$ Dipartimento di Statistica, Informatica, Applicazioni, Università di Firenze, viale Morgagni 59, 50134 Firenze, Italy

E-mail: emanuela.dreassi@unifi.it

${ }^{3}$ School of Mathematical Sciences, University of Nottingham, University

Park, Nottingham, NG7 2RD, UK

E-mail: fabrizio.leisen@gmail.com

${ }^{4}$ Accademia Navale, viale Italia 72, 57100 Livorno, Italy

E-mail: pratel@mail.dm.unipi.it

${ }^{5}$ Dipartimento di Scienze Statistiche "P. Fortunati", Università di Bologna, via delle Belle Arti 41, 40126 Bologna, Italy

E-mail: pietro.rigo@unibo.it 